\title{
50-jährige Patientin mit Sodbrennen
}

\section{0-year-old woman with heartburn}

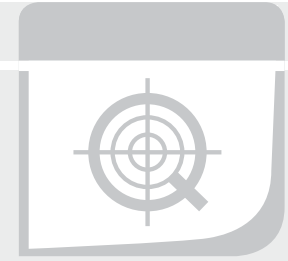

\author{
Autoren \\ Klaus Muehlenberg, Claudia Metzler, Oliver Pech \\ Institut \\ Klinik für Gastroenterologie und interventionelle Endoskopie, \\ Krankenhaus Barmherzige Brüder, Regensburg \\ Bibliografie \\ DOI http://dx.doi.org/10.1055/s-0042-120189 \\ Dtsch Med Wochenschr 2017; 142: 15-16 \\ (c) Georg Thieme Verlag KG Stuttgart · New York \\ ISSN 0012-0472
}

Korrespondenzadresse

Dr. med. Klaus Muehlenberg

Klinik für Gastroenterologie und interventionelle Endoskopie,

Krankenhaus Barmherzige Brüder

Prüfeninger Straße 86

93049 Regensburg

klaus.muehlenberg@barmherzige-regensburg.de

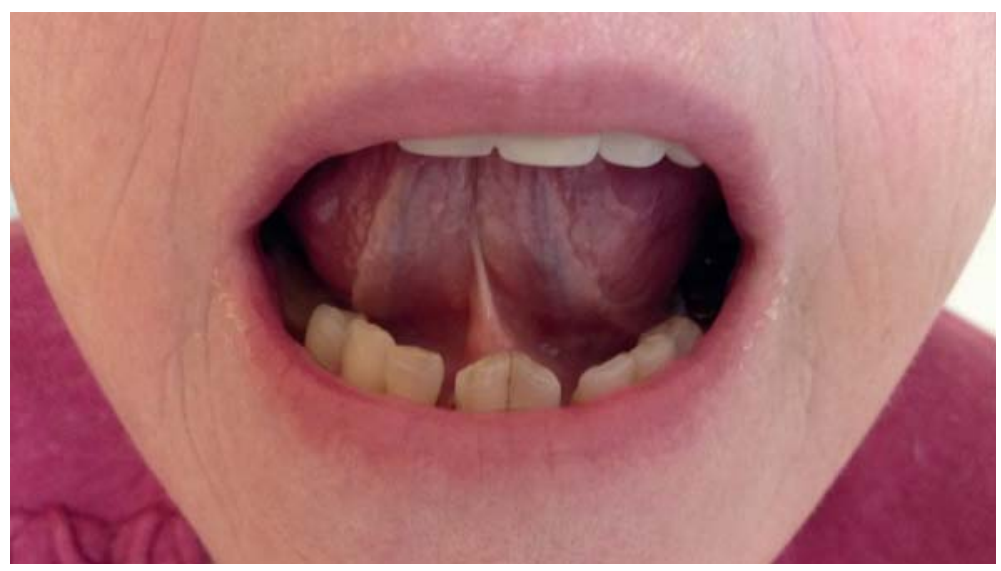

Abb. 1 Mund und Zungengrund.

Eine 50-jährige Patientin leidet unter ausgeprägtem Sodbrennen. Ihr Hausarzt schlägt eine laparoskopische Fundoplikatio zur Behebung der Beschwerden vor. Zuvor sind eine 24-Stunden-pH-Metrie, Gastroskopie und Ösophagusmanometrie als präoperative Untersuchungen geplant. Bei der Magenspiegelung fällt bereits der Endoskopieschwester auf, dass der Beißring (Plastikschutz für das Endoskop) kaum zwischen die Zähne einzuführen ist. Die Inspektion des Mundes und der Mundhöhle weist 2 pathologische Befunde auf.

\section{FRAGEN}

? Welche Befunde sind es?

? Erlauben diese Befunde eine Diagnose? Wenn ja, welche?

? Sind Differenzialdiagnosen möglich? Wenn ja, welche? 


\section{0-jährige Patientin mit Sodbrennen}
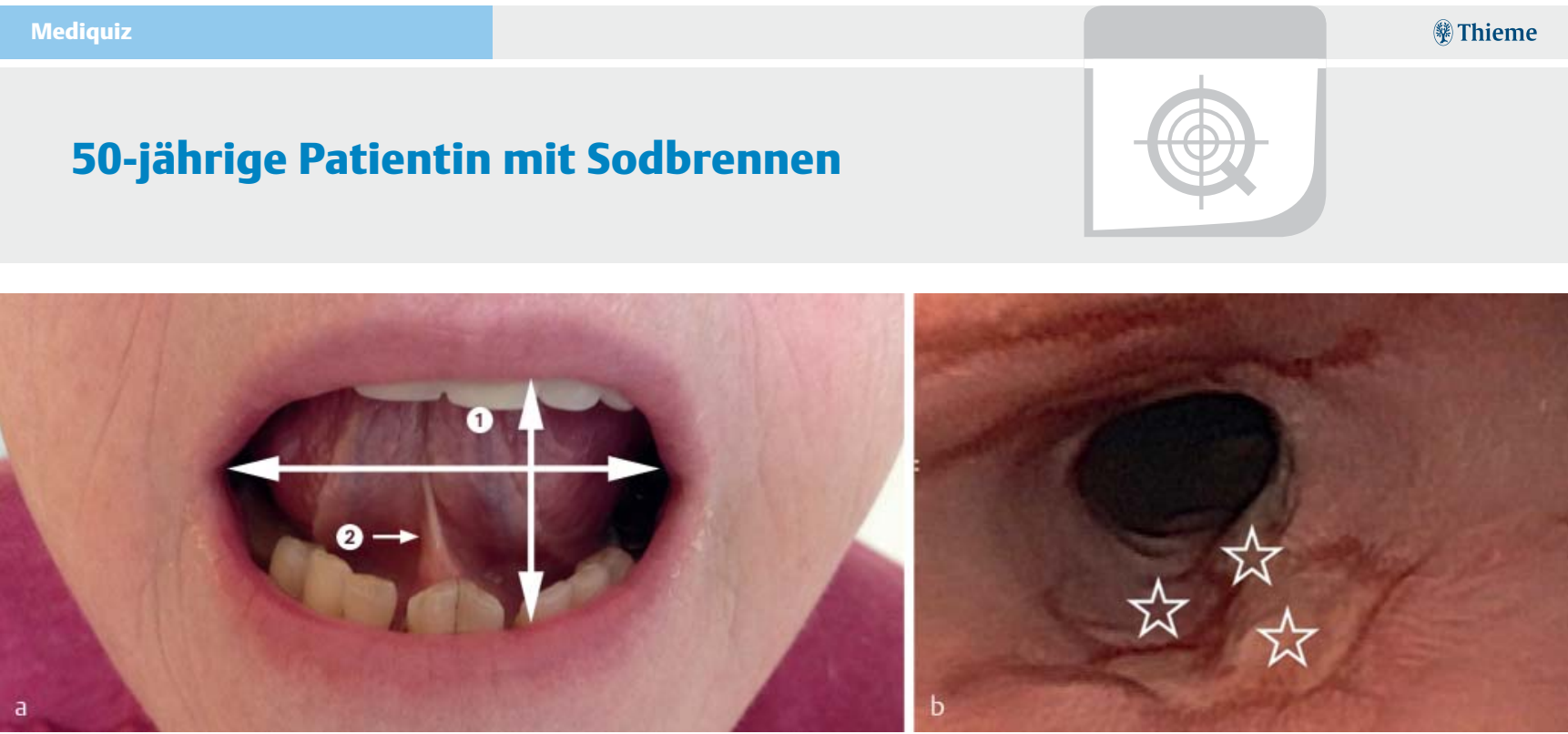

Abb. 2 Mund und Zungengrund a. Endoskopisches Bild des aboralen Ösophagus b.

\section{Befunde}

1. Mikrostomie

2. verkürztes Zungenbändchen

\section{Diagnose}

- Systemische Sklerose mit gastrointestinaler Beteiligung (Refluxösophagitis).

\section{Erläuterung}

Die Systemische Sklerose dieser Patientin war bislang nicht erkannt worden. Erst die genauere Inspektion mit wachsartig starrer Haut (Madonnenfinger, Maskengesicht), die gezielte Anamnese und der Nachweis von SCL-70-Antikörpern führten zweifelsfrei zur Diagnose dieser mit einer Inzidenz von 2-3/ 100000 selteneren rheumatischen Systemerkrankung [1]. Das Sodbrennen hatte die Patientin lange hingenommen, ebenso wie das Abblassen ihrer Finger, die Kälteempfindlichkeit und Blauverfärbung der Hände (Raynaud-Syndrom) sowie intermittierende Durchfälle. Gastrointestinale Symptome sind bei der systemischen Sklerose häufig [2].

- Tab. 1 Beteiligung des Magen-Darm-Traktes bei Systemischer Sklerose in Prozent [2].

\begin{tabular}{|l|l|}
\hline Ösophagus & $75-90 \%$ \\
\hline Magen & $50 \%$ \\
\hline Dünndarm & $40-70 \%$ \\
\hline Kolon & $20-50 \%$ \\
\hline anorektal & $50-70 \%$ \\
\hline
\end{tabular}

Infolge der Fibrosierung und obliterierender Angiopathie kommt es nicht nur zu Hautveränderungen, sondern auch zu Motilitätsstörungen des Magen-Darm-Traktes mit Sodbrennen, Schluckstörungen, Durchfall oder Obstipation ( $\vee$ Tab. 1). Die Fibrosierung der Speiseröhrenmuskulatur führt zu einer „starren“ Speiseröhre und mangels genügender Peristaltik des Ösophagus (fehlende Clearance) zu einer Refluxösophagitis ( $\bullet$ Abb. 2, Sterne).

Veranlasst wurden Untersuchungen anderer bei Systemischer Sklerose potenziell befallener Organe (Niere, Herz, Lunge) und damit die Entscheidung, ob eine antientzündliche (Hydroxychloroquin) bzw. immunsuppressive Therapie (z. B. Azathioprin, Cyclophosphamid oder Mycophenolat-Mofetil) erforderlich war [3]. Aufgrund der kutanen und gastrointestinalen Manifestation erhielt die vorgestellte Patientin Hydroxychloroquin.

Die vorgesehene laparoskopische Fundoplikatio erfolgte nicht, da durch die Operation die zugrundeliegende Motilitätsstörung nicht beseitigt worden wäre. Behandelt wurde die Refluxösophagitis mit Protonenpumpenhemmern und der Vermeidung refluxfördernder Faktoren (Alkohol, Nikotin, Kaffee, Süßes, Südfrüchte).

Literatur

1 Sticherling M. Systemic sclerosis - dermatological aspects. Part 1: Pathogenesis, epidemiology, clinical findings. J Dtsch Dermatol Ges 2012; 10 : $705-718$

2 van den Hoogen F, Khanna D, Fransen J et al. 2013 classification criteria for systemic sclerosis: an American College of Rheumatology/European League against Rheumatism collaborative initiative. Arthritis Rheum 2013; 65: $2737-2747$

3 Denton CP, Hughes M, Gak N et al. BSR and BHPR guideline for the treatment of systemic sclerosis. Rheumatology (Oxford) 2016; 55: 1906-1910 